\title{
Conductors for optics
}

\author{
New research suggests that graphene is not a 'miracle material' for metamaterials and plasmonics. \\ Nature Photonics spoke to Philippe Tassin about what makes a good conductor for these applications.
}

Why has there been so much interest in graphene plasmonics recently?

There is of course a lot of excitement in the physics community about graphene, owing to its wide range of extraordinary electrical, optical and mechanical properties. Both plasmonics and metamaterials typically use metals as conductors. We, and also several other groups, began thinking about how to replace these metals with graphene. I believe there are three good reasons to try using graphene in plasmonic and metamaterial applications. First, graphene is a very thin, atomic-scale system - a characteristic that may allow the further miniaturization of graphene-based devices. Second, graphene can be easily tuned, unlike metals. By applying a gate voltage, you can change the Fermi level of graphene and therefore tune its properties. The conductivity and plasma frequency of a metal are fixed, but with graphene you can change the conductivity at the frequency of operation. The third reason is that metals suffer from significant losses. Even silver and gold, which are believed to be the lowest-loss materials with useful conductivities at optical wavelengths, have high absorption coefficients and correspondingly short plasmon propagation distances when used in metamaterial applications. Graphene is an excellent conductor, and one might therefore hope to reduce losses by using graphene instead of conventional conductors.

\section{What makes a good material for} plasmonics and metamaterials?

In our initial simulations into the use of graphene as a conductor in common metamaterial structures, we didn't see optical resonances. That brought us to the question: what is a good conductor for these applications? First of all, it is important to emphasize that a good material for plasmonics is not necessarily the same as a good material for metamaterials. The real part of the conductivity, which is associated with the 'in phase' part of the current, can dissipate, but the imaginary part of the conductivity, which is related to the 'out of phase' or reactive current, doesn't dissipate. There is a trade-off between high current (and strong optical response) and dissipation. For metamaterials, a small real part of the

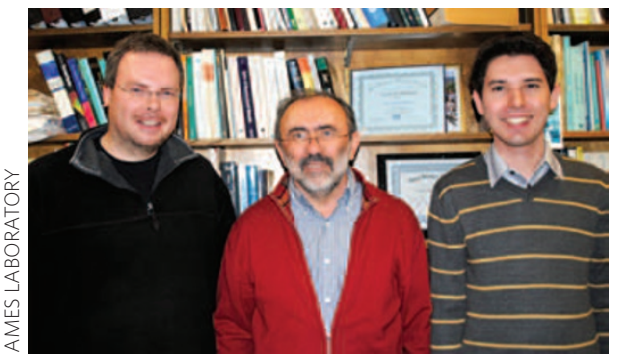

Recent work by Thomas Koschny, Costas Soukoulis, Philippe Tassin and Maria Kafesaki (not pictured) investigates the use of graphene as a conductor for metamaterial and plasmonic applications.

conductivity leads to less dissipation. On the other hand, low conductivity provides only a small current, dipole moment and optical response. We developed a general model for metamaterials with a single resonance in the magnetic dipole response. We then looked at how the material properties affect the overall macroscopic absorption. In our work, we describe the macroscopic absorption as a function of the material's properties. The final figure of merit comes down to the real part of the optical resistivity, which is actually a mixed function of both the imaginary and real parts of the conductivity. There is an additional complication, however, because the thickness of a sample can affect these parameters. Graphene is essentially a zerothickness two-dimensional sample, and this must be taken into account in the figure of merit. This is especially important if you want to compare a two-dimensional conductor like graphene to a bulk three-dimensional conductor such as gold or silver.

\section{Which materials are the best?}

There is no simple answer to this question. Every application has its own requirements for a conducting material. For metamaterials we want a strong optical response to create dipole moments so large that they change the macroscopic material response. The conductors needed for such resonant metamaterials typically benefit from a conductivity that has a large real part, to obtain large resonant currents. On the other hand we also prefer a small imaginary part of the conductivity, which helps to avoid saturation of the resonance frequency and aids scaling towards higher frequencies. The optical resistivity should be as small as possible for metamaterials. We calculated the optical resistivity of noble metals, transition metals, alkali metals, conducting oxides, graphene and others. For microwaves through to visible wavelengths, silver turned out to be the best conductor for metamaterials. Copper is almost as good as silver in the microwave regime, and is easier to work with.

Graphene is ineffective for use in metamaterials, in agreement with our simulations of graphene metamaterials. The conductivity of graphene is orders of magnitude too low to make a resonant metamaterial. People often say that the conductivity of graphene is very high, but this value is achieved by dividing the sheet conductivity by the thickness to obtain a large 'bulk' conductivity. Reducing losses in plasmonic devices - from the point of view of maximizing propagation distance requires materials that have some imaginary part of the conductivity but a small real part of the conductivity. Although graphene may allow very strong confinement at mid-infrared frequencies, the loss would be correspondingly dramatic. Noble metals provide much lower losses for surface plasmon polaritons.

Can you escape the trade-off between confinement and losses?

For a given conductor, I would say no. Achieving better confinement requires more of the field to be dissipated in the metal. There are some ways of tuning a waveguide's geometries to provide small improvements for various designs. Some optimization can be achieved, but there is no significant way to escape the trade-off between confinement and propagation distance. Researchers in our laboratory looked at ways to optimize metamaterial structures - there is a little to be gained through this approach, but it is not very significant. This fact underlines the importance of studying the influence of the conducting material's properties.

\section{INTERVIEW BY DAVID PILE}

Philippe Tassin and co-workers have an Article on graphene as a conductor for metamaterials and plasmonics on page 259 of this issue. 